\title{
MAGNETIC ORDER AT SURFACES AND ELECTRON CAPTURE SPECTROSCOPY (ECS) *
}

\author{
Carl RAU \\ Sektion Physik, University of Munich, D.8000 Munich 40, Fed. Rep. Germany
}

The surface magnetic order (SMO) of ferromagnetic metals is determined with electron-capture spectroscopy (ECS), a novel method which is sensitive to the SMO existing at the topmost atomic layer of a surface. With ECS it is found that the SMO for 3d-transition metals depends drastically on surface orientation; no magnetic dead surface layers are detected. At $\mathrm{Ni}(h k l)$ surfaces "local" SMO exists even at $2 T_{\mathrm{Cb}}$. For Gd surfaces the SMO is quite different from bulk behaviour. Long-range SMO exists for $\mathrm{Gd}$ beyond the bulk Curie temperature $T_{\mathrm{Cb}}$. At the $(100) c(2 \times 2)$ surface of bulk antiferromagnetic Cr long-range SMO is found below the bulk Néel temperature $T_{\mathrm{Nb}}$.

\section{Introduction}

In the past decade much experimental and theoretical work has been devoted towards a deeper comprehension of the magnetic properties of surfaces of ferromagnetic materials (FM) (e.g. Ni, Co, Fe) which play a pivotal role in technologically important fields such as catalysis and corrosion. For a discussion on the interrelation between catalytic and magnetic properties of FM we refer to ref. [1].

The surface magnetic order (SMO) of FM is well characterized by the electron spin polarization (ESP) [2].

Here we report on experimental results on the ESP of surface electrons using electron capture spectroscopy (ECS) which is sensitive to the magnetic state of the topmost atomic layer of a surface.

With ECS not only the "long range" or macroscopic SMO can be investigated but also the "local" or microscopic SMO existing on an atomic scale within a few atomic neighbours. With ECS for the first time the SMO at atomically clean and flat surfaces of single crystals can be investigated. Thereby new and surprising results can be obtained. For bulk antiferromagnetic $\mathrm{Cr}$, e.g., which is widely used as catalyst - such as $\mathrm{Ni}$, Co and $\mathrm{Fe} \mathrm{-} \mathrm{it} \mathrm{is} \mathrm{found} \mathrm{that} \mathrm{long-range} \mathrm{ferromagnetic}$ order exists at the topmost atomic layer of the (100)surface. LEED measurements at this surface show a $c(2 \times 2)$ surface structure due to magnetic surface reconstruction.

\section{Principles of the ECS technique}

The basic process in ECS is the capture of one or two spin-polarized electrons during small-angle reflection of fast deuterons at single crystalline surfaces of magnetic crystals [3,4]. Fig. 1 illustrates an ion trajectory during surface reflection. For a reflection angle $\alpha=0.2^{\circ}$, e.g., a few hundred surface atoms contribute successively in small angle collisions to the specular * Supported by the Bundesministerium für Forschung und
Technologie, Fed. Rep. Germany.

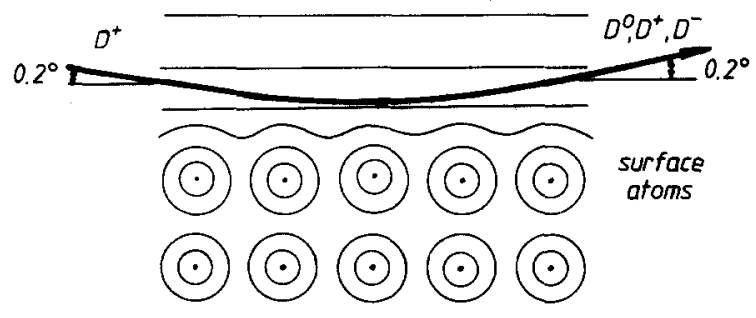

Fig. 1. Scheme of the ion trajectory for a reflection angle $\alpha$ of $0.2^{\circ}$ and surface potential plotted on a plane perpendicular to the reflecting surface.

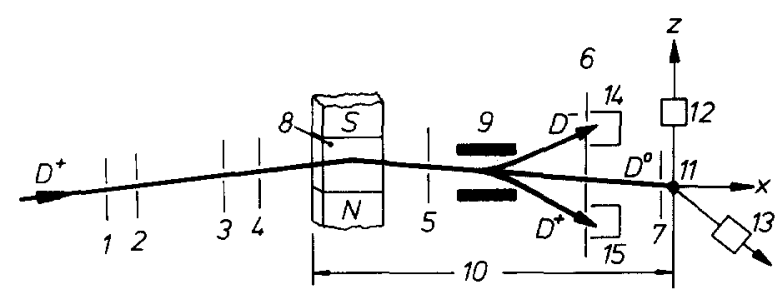

Fig. 2. Experimental arrangement: (1)-(7) ion beam collimating slits; (8) ferromagnetic target $\left(12 \times 8 \times 1.5 \mathrm{~mm}^{3}\right)$, magnetized perpendicular to the incoming beam direction; (9) electrostatic condensor; (10) weak magnetic field $(0.8 \mathrm{mT})$, parallel to the target magnetizing field used as quantization axis ( $+z$ direction) for OEC; (11) T-Ti-target; $(12,13) \alpha$-solid-state detectors; $(14,15)$ Faraday cups.

reflection of the beam. The distance of closest approach $d_{\min }$ of the ion with energy $E_{0}=150 \mathrm{keV}$ is well characterized by the energy component $E_{\perp}$ normal to the surface $\left(E_{\perp}=E_{0} \sin ^{2} \alpha=E_{0} \alpha^{2}=1.8 \mathrm{eV}\right)$. Using interaction potentials which are derived from planar channeling data [5], $d_{\min }$ amounts to $0.2 \mathrm{~nm}$. Therefore the ions probe in real space only the exponential tail of the electronic wave function at the surface. This reveals the extreme surface sensitivitity of ECS. Fig. 2 gives a schematic representation of the experimental arrangement: 
2.1. Detection of long-range SMO using one-electron capture (OEC) processes $\left(D^{+}=e^{-}=D^{0}\right)$

A well collimated deuteron beam (half angle of divergence $<0.025^{\circ}$ ) of $150 \mathrm{keV}$ deuterons is reflected and thereby partially neutralized at a $(h k l)$-surface of a ferromagnetic (or antiferromagnetic) single crystal magnetized to saturation parallel to the surface plane. After reflection the beam passes through an electric field to extract positive and negative charged ions. Therefore only neutralized particles impinge on a $\mathrm{T}$-target and provide $\alpha$-particles via the $\mathrm{T}(\mathrm{d}, \mathrm{n}) \alpha$-reaction. During an adiabatic transition from the high-magnetic field region at the magnetic surface into a weak magnetic field the ESP in the $\mathrm{D}^{0}$-electron shell is partially converted into a nuclear polarization by hyperfine interaction. The nuclear polarization which serves as a measure of the ESP is determined via the T-reaction using the $\alpha$-count ratio $r=N_{z} / N_{y}$ in two $\alpha$-solid state detectors (12, 13 in fig. 2). Defining $P$ along the $+z$-direction (see fig. 2 ) we obtain

$P=\left(n^{+}-n^{-}\right) /\left(n^{+}+n^{-}\right)$,

where $n^{+}$and $n^{-}$represent fractional numbers of electrons with spin moment antiparallel (majority spin electrons) and parallel (minority spin electrons), respectively, to the target magnetizing field. The ESP is directly related to $r[3]$ :

$P=12(1-r) /(r+2)$.

The target magnetizing field is applied to align randomly oriented Weiss domains thereby producing a macroscopic magnetization which defines a preferred direction in space along which the sign and magnitude of $P$ can be measured.

\subsection{Detection of "local" SMO using two-electron capture (TEC) processes $\left(D^{+}+2 e^{-}=D^{-}\right)$}

A beam of $100 \mathrm{keV}$ deuterons is reflected at a demagnetized surface of a ferromagnetic crystal. After reflection the beam passes through an electric field to split spatially the $\mathrm{D}^{-}$and the $\mathrm{D}^{+}$-ions. The $\mathrm{D}^{-}$-ion and the $\mathrm{D}^{+}$-ion currents are then simultaneously detected using two Faraday cups (14, 15 in fig. 2). The basic idea of the TEC experiment - which probes "local" SMO (spin-spin correlations) on an atomic scale - depends on the fact that TEC occurs within one single magnetic domain in which two electrons captured by a single deuteron always possess the same spin alignment given by the direction of the spontaneous ferromagnetic order existing in each domain also in demagnetized samples below $T_{C}$. Two electrons in a domain have either parallel or antiparallel spin orientations corresponding to a triplet or singlet final state in a formed $\mathrm{D}^{-}$-ion. We note that for $\mathrm{D}^{-}$-ions only singlet $-1 s^{2}$-states exist [4]. The formation of $D^{-}$-ions is there- fore drastically suppressed using ferromagnetic surfaces with $P= \pm 100 \%$. At nonmagnetic surfaces (such as $\mathrm{Cu}$ ) with $P=0$ the formation of $D^{-}$-ions is not suppressed. The charge state ratio $R=D^{-} / D^{+}=\left(D^{-}\right.$-fraction of the reflected beam $/ D^{+}$-fraction of the reflected beam) at $\mathrm{Ni}$ and $\mathrm{Cu}$ surfaces is directly related to the corresponding fractional numbers $n_{\mathrm{Ni}}$ and $n_{\mathrm{Cu}}$ for $\mathrm{Ni}$ and $\mathrm{Cu}$ surfaces [4]

$R_{\mathrm{Ni}} / R_{\mathrm{Cu}}=n_{\mathrm{Ni}} / n_{\mathrm{Cu}}$.

With $P=0$ for $\mathrm{Cu}$ and $n_{\mathrm{Cu}}^{+}+n_{\mathrm{Cu}}^{-}=1$ we obtain from eq. (1) $n_{\mathrm{Cu}}^{+}=n_{\mathrm{Cu}}^{-}=n_{\mathrm{Cu}}=0.5$. For $\mathrm{Ni}$ the negative ion formation rate determining fractional electron number is $n_{\mathrm{Ni}}=(1-|P|) / 2$ with $n_{\mathrm{Ni}}^{+}+n_{\mathrm{Ni}^{-}}^{-}=1$. We then yield for the "local" ESP

$\left|P_{\mathrm{Ni}}\right|=1-\left(R_{\mathrm{Ni}} / R_{\mathrm{Cu}}\right)$.

\section{Results and discussion}

\subsection{3d-transition metal surfaces}

\subsubsection{Long-range SMO}

Amongst the $3 \mathrm{~d}$-transition metals $\mathrm{Ni}$ has received the most attention over the past ten years. Table 1 lists ESP results which are obtained at $293 \mathrm{~K}$ with ECS using $\mathrm{OEC}$ at single-crystalline $\mathrm{Ni}$ surfaces. Important characteristics about the experimental data are:

(1) $P=P(h k l)$ : the ESP depends drastically on the surface orientation with respect to both sign and magnitude revealing the importance of measuring ESP as a function of the surface indices $(h k l)$.

(2) $P \neq 0$ at $\mathrm{Ni}(h k l)$ surfaces: these findings show that at these surfaces no intrinsic ferromagnetic dead layers exist and reveal the inapplicability of theoretical models in which the possible existence of these dead layers is investigated [6]. In table 1 we have also listed theoretical $P(h k l)$ values for Fermi electrons which are derived from one-dimensional spin-polarized electron densities of a recent bulk electron band structure calculation [7]. To this we note that in the grazing-angle ECS experiment only the most weakly bound surface electrons (Fermi electrons) are available for capture $[3,8]$. The perfect agreement concerning the sign of the re-

Table 1

Comparison of Ni-ESP data for Fermi electrons as deduced from band structure calculations [7,9] and from ECS experiments using $\mathrm{OEC}$

\begin{tabular}{llll}
\hline$(h k l)$ & \multicolumn{2}{l}{ Band structures: $P(\%)$} & \multirow{2}{*}{$\begin{array}{l}\text { ECS experiments: } P(\mathscr{F}) \\
\text { surface-ESP; OEC }\end{array}$} \\
\cline { 2 - 3 } & bulk-ESP & surface-ESP & \\
\hline 110 & $-81[7]$ & & -96 \\
100 & $-94[7]$ & & -65 \\
100 & $-78[9]$ & $-57[9]$ & \\
111 & $-75[7]$ & & -44 \\
120 & $+17[7]$ & & +16 \\
\hline
\end{tabular}




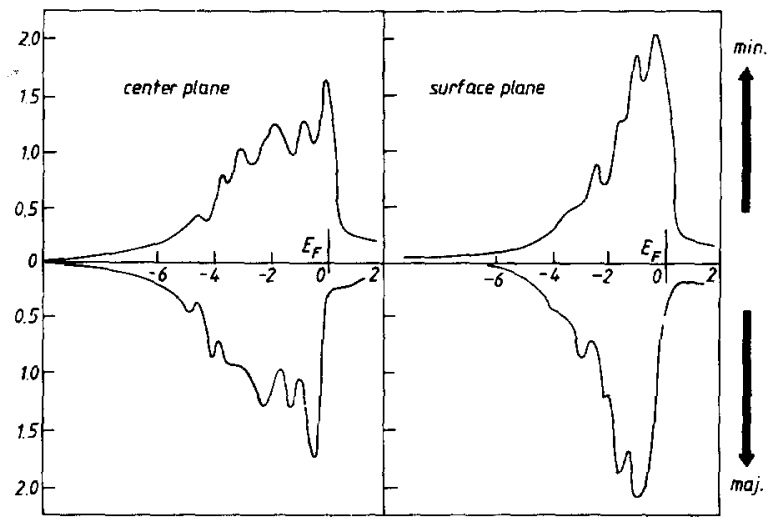

Fig. 3. Self-consistent planar, spin-polarized electron densities for the center plane and the surface plane of $\mathrm{Ni}(100)$ : Electrons/eV/atom/spin as function of electron energy $E$ in $\mathrm{eV}$ according to ref. [9]. In the upper part of fig. 3 the minority-spin densities, and in the lower part of fig. 3 the majority-spin densities are plotted.

spective $P(h k l)$ values indicates that for $\mathrm{Ni}$ there are no drastic changes in the magnetic structure in going from the bulk to the surface.

Quite recently for $\mathrm{Ni}(100)$ self-consistent surface band structure calculations have become available where the influence of surface states (SS) on the SMO is investigated and which show that there are no drastic changes in the magnetic structure in going from the bulk to the surface. Fig. 3 shows spin-polarized planar electron densities for the center plane and the surface plane of a 9 layer thin $\mathrm{Ni}(100)$ film which were calculated by Wang and Freeman [9]. These authors find majority-SS at the Fermi level which reduce the minority-ESP of the Fermi electrons from $-78 \%$ (center plane) to $-57 \%$ (surface plane) in good agreement with the ECS experiment (see also table 1).

\subsection{2. "Local" $S M O$}

The first ECS experiments using TEC we performed at demagnetized $\mathrm{Ni}(110)$ surfaces. From table 1 we see that the magnetized (110)-surface of $\mathrm{Ni}$ at $293 \mathrm{~K}$ exhibits an ESP of $-96 \%$ using OEC showing that the tail of the electron density at this surface overwhelmingly

Table 2

Experimental data on the "local" and long-range ESP at $\mathrm{Ni}(h k l)$ surfaces

\begin{tabular}{lllll}
\hline & ECS & & & \\
\cline { 2 - 5 } & TEC & & OEC \\
\hline$(h k l)$ & $R_{\mathrm{Ni}} \times 10^{3}$ & $R_{\mathrm{Cu}} \times 10^{3}$ & $|P|(\%)$ & $P(\%)$ \\
$(110)$ & 0.35 & 5.70 & 94 & -96 \\
$(100)$ & 1.45 & 5.25 & 72 & -65 \\
$(111)$ & 3.05 & 5.75 & 47 & -44 \\
$(120)$ & 4.62 & - & 14 & +15 \\
polycr. & - & 5.37 & & \\
\hline
\end{tabular}

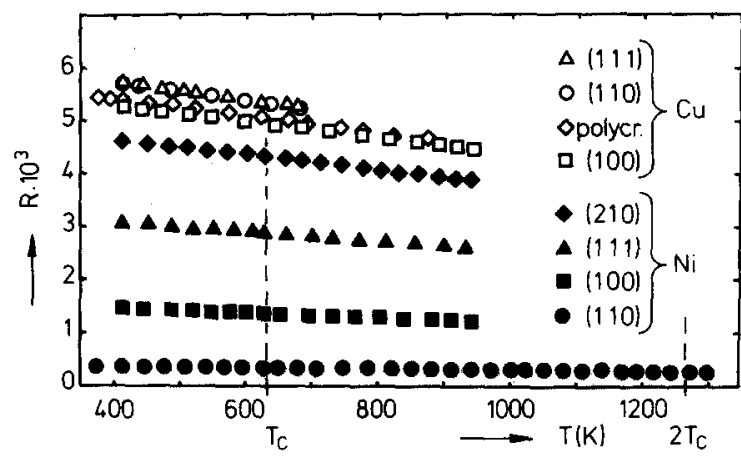

Fig. 4. Charge state ratios $R_{\mathrm{Ni}}(h k l)$ and $R_{\mathrm{Cu}}(h k l)$ as a function of the target temperature $T$. Polycryst. refers to polycrystalline $\mathrm{Cu}$ which is used to evaluate $\mid P_{\mathrm{Ni}}(120 \mid$. For $\mathrm{Ni}$ only each second measuring point is drawn to give a clear pot.

(98\%) consists of electrons of one spin direction only.

Reflecting $100 \mathrm{keV}$ deuterons at $\mathrm{Ni}(110)$ and $\mathrm{Cu}(110)$ surfaces at $293 \mathrm{~K}$ and using TEC drastically different charge state ratios are obtained. $R_{\mathrm{Cu}}(110)$ amounts to $5.7 \times 10^{-3}$ whereas $R_{\mathrm{Ni}}(110)$ amounts only to $0.35 \times$ $10^{-3}$. From eq. (4) we obtain $\left|P_{\mathrm{Ni}}(110)\right|=94 \%$ which is close to the long-range ESP measured with OEC.

The results of further systematic studies [4] at various $\mathrm{Ni}(h k l)$ surfaces are listed in table 2 together with OEC data. From the comparison of the respective ESP

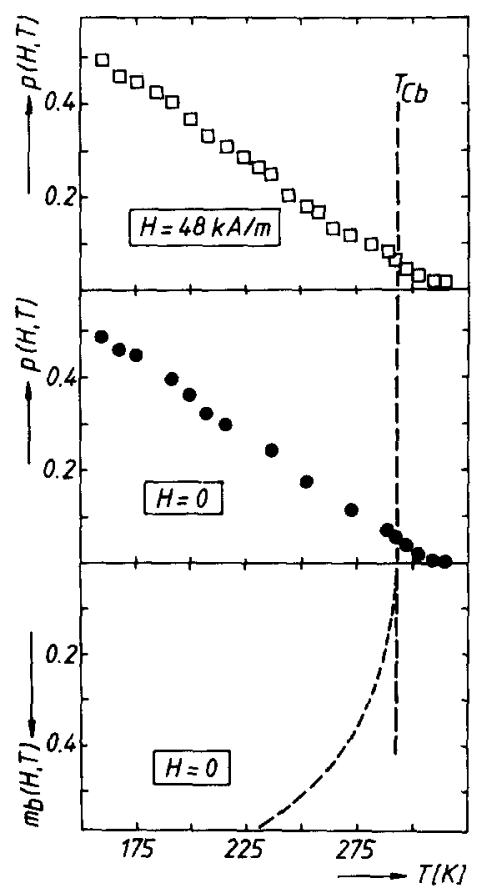

Fig. 5. Upper part: reduced $\operatorname{ESP} p(H, T)$ at Gd surfaces as a function of temperature $T$ measured in a magnetizing field of $H=48 \mathrm{kA} / \mathrm{m}$ with ECS using OEC. Middle part: $p(H, T)$ as a function of $T$ for $H=0$ (see text and ref. [14]. Lower part: reduced spontaneous magnetization $m_{b}(0, T)$ of bulk $G d$ as a function of $T$. 


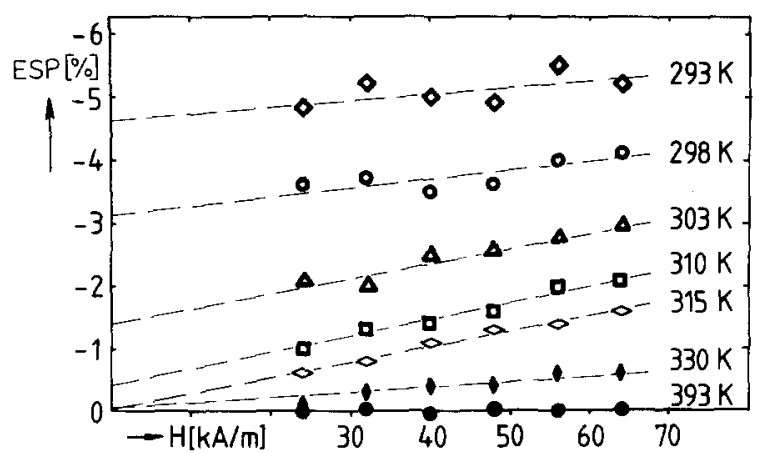

Fig. 6. ESP isotherms (293-393 K) at Gd surfaces as a function of the applied magnetic field $H$.

values, both measured at $293 \mathrm{~K}$, we see that TEC experiments open a new and simple way to investigate SMO.

Further experiments where the temperature dependence of the "local" SMO at $\mathrm{Ni}(h k l)$ surfaces is investigated are shown in fig. 4 . From this we see there are no drastic changes in the "local" SMO for temperatures far above the ferromagnetic bulk Curie temperature $T_{\mathrm{Cb}}$ of $\mathrm{Ni}$ indicating that "local" SMO at $\mathrm{Ni}$ surfaces exists on an atomic scale even at $2 T_{\mathrm{Cb}}$. These findings suggest that a "local" nearly temperature-independent ferromagnetic exchange splitting - which is discussed in the "local" band picture [10-13] - exists even at these high temperatures.

\subsection{Magnetic surface phase transitions}

The influence of a surface on magnetic phase transitions has been investigated within the frame of different theoretical models (see ref. [13] and refs. therein). There is considerable interest in experimental data on the temperature dependence of the SMO. One of the reasons for the lack of experimental data is that it is difficult to prepare clean surfaces which remain uncontaminated and well-defined during the multiple heating and cooling cycles which are necessary to obtain reliable information.

Among the ferromagnetic metals the rare earth metal Gd with $T_{\mathrm{Cb}}$ around room temperature is a good candidate because at room temperature the bulk impurity diffusion is negligible and undesirable gas adsorption can be neglected under extreme UHV conditions. The upper part of fig. 5 shows the reduced $\operatorname{ESP} p(H$, $T)=P(H, T) / P(H, 0)$ measured with OEC at atomically clean polycrystalline $\mathrm{Gd}$ surfaces in a magnetic field $H=48 \mathrm{kA} / \mathrm{m}$. In the middle part of fig. $5 p(H$, $T$ ) is plotted for $H=0$. These values are derived from a linear extrapolation of isotherm $p(H)$ data towards $H=0$ according to ref. [14]. For comparison the lower part of fig. 5 gives the behavior of the reduced bulk spontaneous magnetization $m_{\mathrm{b}}(H, T)=M_{\mathrm{b}}(0$,

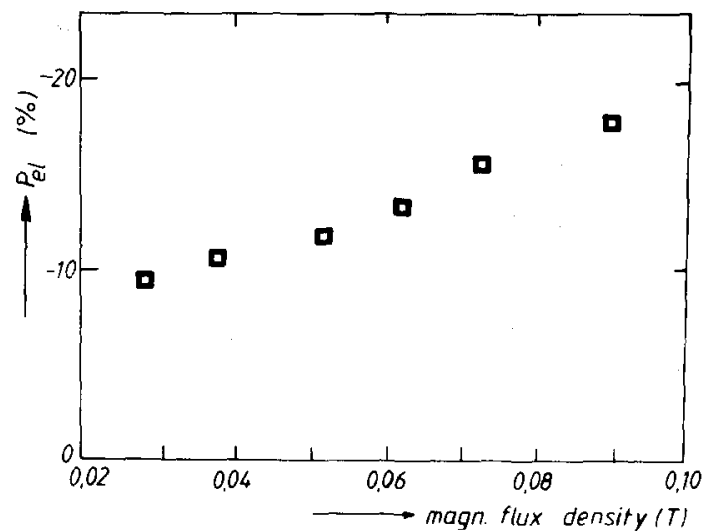

Fig. 7. ESP at $\operatorname{Cr}(100) c(2 \times 2)$ surfaces as a function of the applied magnetic field.

$T) / M_{\mathrm{b}}(0,0)$. Fig. 6 shows $P(H)$ isotherms for $T>T_{\mathrm{Cb}}$ $=292.5 \mathrm{~K}$ which is measured in situ by an inductive method. Three interesting aspects are to be noted [15]:

(1) $P \neq 0$ below $T_{\mathrm{Cb}}$ agrees well with the RKKY theory and with ferromagnetic band structure calculations of Harmon and Freeman [16] which predict a minority-ESP of Fermi electrons for all calculated lowindex $(h k l)$ surface orientations.

(2) The temperature dependence of $p$ at the topmost surface layer is drastically different from bulk behaviour (see fig. 5). Between $160 \mathrm{~K}$ and approximately $300 \mathrm{~K}$ the ESP changes linearly with $T$.

(3) $P \neq 0$ for $H=0$ above $T_{\mathrm{Cb}}$ shows that the surface Curie temperature $T_{\mathrm{Cs}}$ is at least $15 \mathrm{~K}$ beyond $T_{\mathrm{Cb}}$ indicating that the surface layer has a highly anisotropic exchange where the surface coupling constant is larger than the bulk exchange coupling constant $[17,18]$. We also remark that already a change of the lattice constant at the surface by a few per cent could increase the magnetic exchange constant by a factor of two.

\subsection{Surfaces of antiferromagnetic chromium}

Metallic $\mathrm{Cr}$ is antiferromagnetic below the bulk Néel temperature $T_{\mathrm{Nb}}=310 \mathrm{~K}$ and consists of two compensating ferromagnetic sublattices producing zero bulk magnetization. The spin structure in the (100)-direction is sinusoidal and incommensurate with the lattice period suggesting ferromagnetic order at the topmost atomic (100)-surface layer. Fig. 7 gives a plot of long-range ESP measured at $\operatorname{Cr}(100)$ at $293 \mathrm{~K}$ as a function of the applied magnetic field $B$. For $B=0.09 \mathrm{~T}$ an ESP of $-(18 \pm 2) \%$ is found which clearly shows that below $T_{\mathrm{Nb}}$ long-range $\mathrm{SMO}$ exists at this surface which shows a $c(2 \times 2)$ surface structure as found in the LEED measurement. With increasing temperature the ESP decreases to around a few per cent near $T_{\mathrm{Nb}}$. These findings can serve as a crucial test for the applicability 
of theoretical calculations on the SMO of antiferromagnetic materials [19].

\section{Summary and conclusions}

Electron capture spectroscopy (ECS) is a novel method for magnetic surface analysis with high spatial sensitivity. With ECS the surface magnetic order (SMO) of the topmost atomic layer of a magnetic material can be measured. ECS has been applied to a series of ferromagnetic materials to investigate interesting phenomena. With ECS studies it is shown that only well defined and atomically clean surfaces reflect the magnetic and electronic properties of the material under consideration. Nickel, e.g., exhibits a high electron spin polarization (ESP), well in accordance with predictions of band structure calculations; no so-called intrinsic magnetic dead layers could be observed at uncontaminated surfaces. For Gd it is found that the temperature dependence of SMO is quite different from that of the bulk, a finding most important for the promotion of prevailing theoretical notations. In further ECS studies it is shown that the reconstructed $(100) c(2 \times 2)$ surface of antiferromagnetic bulk $\mathrm{Cr}$ is ferromagnetic. This is shown by ECS in a straightforward way in combination with LEED investigations.

\section{References}

[1] P. Fulde, Physica 91B (1977) 251.

[2] M.C. Gutzwiller, AIP Conf. Proc. 10 (1973) 1179.

[3] C. Rau, Comments Solid State Phys. 9 (1980) 177

[4] C. Rau and S. Eichner, Phys. Rev. Lett. 47 (1981) 939.

[5] I.A. Torrens, Interatomic Potentials (Academic, New York, 1972).

[6] P. Fulde, A. Luther and R.E. Watson, Phys. Rev. B8 (1973) 440 .

[7] E. Marschall and H. Bross, Phys. Stat. Sol. (b)90 (1978) 241.

[8] C. Rau, J. Magn. Magn. Mat. 30 (1982) 141.

[9] C.S. Wang and A.J. Freeman, Phys. Rev. B21 (1980) 4585.

[10] H. Capellmann, J. Phys. F4 (1974) 1466.

[11] J.B. Sokoloff, Phys. Rev. Lett. 31 (1973) 1417.

[12] V. Korenman, J.L. Murray and R.E. Prange, Phys. Rev. B16 (1977) 4032, 4048, 4058.

[13] T. Moriya, J. Magn. Magn. Mat. 14 (1979) 1.

[14] I. Balberg and J.S. Helman, Phys. Rev. B18 (1978) 303.

[15] C. Rau and S. Eichner, to be published.

[16] B.N. Harmon and A.J. Freeman, Phys. Rev. B10 (1974) 1979.

[17] J.S. Helman, private communication.

[18] S. Selzer and N. Majlis, to be published.

[19] G. Allan, Surface Sci. 74 (1978) 79. 